TRANSACTIONS OF THE

AMERICAN MATHEMATICAL SOCIETY

Volume 355, Number 9 , Pages 3513-3535

S 0002-9947(03)03240-9

Article electronically published on April 25, 2003

\title{
PLANAR CONVEX BODIES, FOURIER TRANSFORM, LATTICE POINTS, AND IRREGULARITIES OF DISTRIBUTION
}

\author{
L. BRANDOLINI, A. IOSEVICH, AND G. TRAVAGLINI
}

\begin{abstract}
Let $B$ be a convex body in the plane. The purpose of this paper is a systematic study of the geometric properties of the boundary of $B$, and the consequences of these properties for the distribution of lattice points in rotated and translated copies of $\rho B$ ( $\rho$ being a large positive number), irregularities of distribution, and the spherical average decay of the Fourier transform of the characteristic function of $B$. The analysis makes use of two notions of "dimension" of a convex set. The first notion is defined in terms of the number of sides required to approximate a convex set by a polygon up to a certain degree of accuracy. The second is the fractal dimension of the image of the Gauss map of $B$. The results stated in terms of these quantities are essentially sharp and lead to a nearly complete description of the problems in question.
\end{abstract}

\section{INTRODUCTION}

Suppose $B \subset \mathbb{R}^{2}$ is a convex body: a convex compact set with nonempty interior. Many classical problems in analysis, geometry, and number theory are stated in terms of basic properties of such sets. For example, we may consider the difference between the number of lattice points inside the dilated set $\rho B$ and its area, i.e., the discrepancy

$$
D_{\rho}(B)=\operatorname{card}\left(\rho B \cap \mathbb{Z}^{2}\right)-\rho^{2}|B|,
$$

where $|\cdot|$ denotes the area. Among the many natural questions we can ask about this problem (see the section on lattice points below) is, how does the geometry of $B$ affect the growth rate of the discrepancy function? As we shall see, there are results that do not distinguish among various convex sets. However, we shall also see that the behavior of the above discrepancy functions corresponding to different convex sets may vary dramatically, and that this behavior may be described in terms of natural and readily computable geometric quantities.

The above question on lattice points has a consequence in the study of irregularities of distribution. Suppose $\mathcal{P}=\left\{z_{j}\right\}_{j=1}^{N}$ is a distribution of $N$ points in the unit square $U=[0,1]^{2}$ treated as the torus $\mathbb{T}^{2}$. Let $B$ be a convex body in $U$ with

Received by the editors February 11, 2002.

2000 Mathematics Subject Classification. Primary 42B10; Secondary 52A10.

Key words and phrases. Decay of Fourier transforms, convex bodies, Minkowski dimension, lattice points, irregularities of distribution.

The first and third authors are supported by MURST. The second author is supported by NSF grant DMS00-87339. 
diameter smaller than 1 . Assume $\varepsilon \leq 1, t \in \mathbb{T}^{2}$. Then certain sharp upper estimates for the discrepancy

$$
D(\mathcal{P}, \varepsilon, t)=\sum_{j=1}^{N} \chi_{\varepsilon B-t}\left(z_{j}\right)-N \varepsilon^{2}|B|
$$

can be obtained from related estimates for lattice points (by a suitable trick we shall reduce to the case when $N$ is a square, which in turn is an easy corollary).

At the heart of the lattice point and the irregularities of distribution problems is the Fourier transform of the characteristic function of $B$. Our approach is to study the effect of the geometric properties of $B$ on the decay rate of the Fourier transform of the characteristic function of $B$ and its variants. We shall then use this analysis to obtain precise information about the discrepancy functions described above.

How should we distinguish among the various convex planar sets? The lattice point problem suggests one natural approach. It was observed by Gauss that $D_{\rho}(B) \lesssim \rho$, since the boundary of $B$ is one-dimensional. Consider the case when $B$ is a unit square with sides parallel to the axes. When $\rho$ is an integer, the boundary of $\rho B$ contains $\approx \rho$ integer lattice points, thus showing that this estimate cannot be improved. However, if $B$ is a disc, the boundary of $\rho B$ "curves away" from the integer lattice. In fact, it is known (see [16]) that the estimate for $D_{\rho}(B)$ in this case is much better. These two examples suggest that the curvature of the boundary may be the key distinguishing factor among convex sets. The boundary of the square has no curvature, which leads to a poor discrepancy estimate, whereas the boundary of the disc has everywhere non-vanishing curvature, and the estimate for the discrepancy function is considerably better.

The notion of curvature alluded to in the previous paragraph is the standard geometric, or Gaussian, curvature, defined as the determinant of the differential of the Gauss map that maps each point on the boundary of a convex set to the unit normal at that point. It turns out that the geometric curvature alone does not capture the relevant properties of convex planar sets fully. To see this, let us return to the case of the unit square. While it is true that the discrepancy function is terrible if the sides of the square are parallel to the axes, the discrepancy function becomes practically non-existent, even better than the discrepancy function for the disc, if the square is rotated by a sufficiently irrational angle (see [14]). In fact, it is precisely the "flatness" of a square that keeps its boundary from hitting hardly any lattice points when it is rotated. This suggests that for "most" rotations, convex sets with "flat" boundaries behave better as far as discrepancy functions are concerned.

In this paper we consider the rotated and translated copies $\sigma^{-1}(\rho B)-t$ (where $\sigma \in S O(2), t \in \mathbb{T}^{2}$ ) of the dilated body $\rho B$ (here $\rho$ is a large positive number), and we study the $L^{1}$ mean

$$
\int_{\mathbb{T}^{2}} \int_{S O(2)}\left|D_{\rho}\left(\sigma^{-1}(B)-t\right)\right| d \sigma
$$

of the discrepancy

$$
D_{\rho}\left(\sigma^{-1}(B)-t\right)=\operatorname{card}\left(\left(\rho \sigma^{-1}(B)-t\right) \cap \mathbb{Z}^{2}\right)-\rho^{2}|B| .
$$


The reason for choosing the $L^{1}$ mean among other $L^{p}$ means will be clear soon. Let us also say that in many cases, averaging makes a discrepancy problem easier. For example, the Gauss circle problem is a basic and unsolved problem, while one can obtain (see e.g. [15] or [8]) a sharp result averaging in $L^{2}$ over translations of the discs and using only Parseval's identity and some properties of Bessel functions.

Let us go back to the geometry of $B$. The above observations can be exploited in a number of ways. If "flatness" is good, then the family of rotated copies of $B$, is better if $B$ is close to being a polygon. This means that $B$ is good if it can be approximated by a polygon with relatively few sides (the construction we are going to describe has been studied in [19] and [23, see also [26]). We choose an arbitrary point on the boundary of $B$ and draw a chord to another point on the boundary of $B$ in such a way that the maximum distance from the chord to the boundary of $B$ is $\rho^{-1}$. Roughly speaking, if the number of sides of the above inscribed polygon is $\lesssim \rho^{\alpha}$, we say that the dimension of $B$ is at least $\alpha$ (we shall explain later why for most of the paper we prefer not to consider the infimum of the $\alpha$ 's). Note that $B$ is a polygon if and only if we can choose $\alpha=0$, and if $B$ is a circle, then $\alpha=1 / 2$ works.

We can also take the following "dual" point of view. If $B$ is close to a polygon, then its boundary $\partial B$ has relatively few normals. A more precise way of saying this is that the area of the $\delta$-neighborhood of the image of $\partial B$ under the Gauss map is $\lesssim \delta^{1-d}$. If $B$ is a disc, we can only take $d=1$. On the other hand, we can choose $d=0$ if and only if $B$ is a polygon. As another example, let $B$ be a polygon with infinitely many sides, the normals of which have apertures in the sequence $n^{-\beta}$, $\beta>0$; it is easy to see that in this case we can take $d=(1+\beta)^{-1}$.

Introducing the infima $\alpha^{*}$ and $d^{*}$ (note that $d^{*}$ is the upper Minkowski dimension of the image of the Gauss map), we have $\alpha^{*} \leq d^{*} /\left(d^{*}+1\right)$, and we can also prove that this bound is the best possible. On the other hand, we can show that $\alpha^{*}$ can be as close to 0 as we want, even when $d^{*}$ is away from 0 .

This paper is structured as follows. We shall first describe the main analytic idea, the effect of the geometry of a convex set on the average decay of the Fourier transform of the characteristic function of $B$. We shall also prove that polygons provide the fastest possible decay. We shall then apply our estimates to the distribution of lattice points in convex domains and the problem of irregularities of distribution.

In this paper most of the ideas used to prove the results on the average decay are new, while almost all the applications to lattice points and irregularities of distribution are straightforward.

We conclude the introduction by noting that a notion of a dimension of a convex set may be applicable and natural in a number of interesting problems in analysis and combinatorics. For example, the Falconer distance conjecture says that if the Hausdorff dimension of a planar set is greater than 1, then the set of Euclidean distances among the points of this set has positive Lebesgue measure. However, if the Euclidean distance is replaced by the "taxi-cab" $\left(l^{1}\right)$ metric, the conjecture is clearly false, and in fact the set is required to have Hausdorff dimension 2 before the same conclusion on the distance set is possible. It is reasonable to ask whether distances induced by convex sets with "intermediate dimension" provide examples of intermediate behavior in the Falconer distance problem. We hope to address this and other issues of this type in a subsequent paper. 
1.1. $L^{p}$ average decay of the Fourier transform. The study of the decay of the Fourier transform

$$
\widehat{\chi}_{B}(\xi)=\int_{B} e^{-2 \pi i \xi \cdot x} d x
$$

as $|\xi| \rightarrow \infty$ is a classical subject. When $\partial B$ has strictly positive curvature, then $\left|\widehat{\chi}_{B}(\xi)\right| \lesssim|\xi|^{-3 / 2}$. However, when $\partial B$ contains points where the Gaussian curvature vanishes, then the above inequality is no longer true. For example, when $B$ is a polygon and $\Theta=(\cos \theta \cdot \sin \theta)$, then $\widehat{\chi}_{B}(\rho \Theta)$ decays as $\rho^{-1}$ in some directions and as $\rho^{-2}$ in most directions. In such cases it is useful to study the $L^{p}$ spherical average decay of $\widehat{\chi}_{B}$, given by

$$
\left\|\widehat{\chi}_{B}(\rho \cdot)\right\|_{L^{p}\left(\Sigma_{1}\right)},
$$

where $\Sigma_{1}$ is the unit circle and $1 \leq p \leq \infty$. Here a basic result is Podkorytov's theorem

$$
\left\|\widehat{\chi}_{B}(\rho \cdot)\right\|_{L^{2}\left(\Sigma_{1}\right)} \lesssim \rho^{-3 / 2}
$$

(see [19]), where no regularity assumption on the boundary $\partial B$ is required.

Throughout this paper $X \lesssim Y$ will mean that $X \leq c Y$, with $c$ depending only on the body $B$ under consideration. Moreover, we shall always assume $\rho \geq 2$.

The study of (1.1) turns out to have applications to several problems, such as the distribution of lattice points in large convex domains ([20], [25], [7], [8]), irregularities of distribution ([17, [7]), summation of multiple Fourier expansions ([9], [5, [6]), and estimates for generalized Radon transforms ([21]).

The paper [8] contains the following rather complete study of (1.1) under the additional assumption that $\partial B$ is piecewise smooth. When $p=2,(1.2)$ says that the rate of decay of (1.1) is independent of the shape of $B$. When $2<p \leq \infty$, any order of decay between that of the disc and of a polygon is possible. On the other hand, when $1 \leq p<2$, a convex body with piecewise smooth boundary behaves either like a disc or like a polygon. In particular, when $P$ is a polygon we have the sharp bound

$$
\left\|\widehat{\chi}_{P}(\rho \cdot)\right\|_{L^{1}\left(\Sigma_{1}\right)} \lesssim \rho^{-2} \log \rho
$$

and when $B$ has piecewise smooth boundary, but is not a polygon, we have the sharp bound

$$
\left\|\widehat{\chi}_{B}(\rho \cdot)\right\|_{L^{1}\left(\Sigma_{1}\right)} \lesssim c \rho^{-3 / 2} .
$$

Actually, (1.4) is sharp whenever $\partial B$ contains at least one point where the Gaussian curvature exists and is different from zero.

The above dichotomy, pointed out in [8, is no longer valid for arbitrary convex bodies. The existence of "chaotic" decays has been pointed out in [8, p. 553], using an abstract argument on convex sets. Unfortunately, that argument is not constructive, nor does it provide nontrivial explicit bounds for the average decay.

The main analytic tool of this paper is the $L^{p}$ average decay for arbitrary convex planar bodies when $1 \leq p \leq 2$. In essence, we shall consider the $L^{1}$ average decay and the $L^{2}$ average decay. The results for intermediate exponents can be essentially obtained by interpolation. Roughly speaking, the $L^{2}$ average decay is an "all cats are grey in the dark" phenomenon, where the decay does not distinguish among the different convex bodies. On the other hand, the $L^{1}$ average decay determines, in a sense, how close a convex set is to a polygon. 
1.2. Inscribed polygons. We introduce the following notation. For any $\Theta=$ $(\cos \theta, \sin \theta)$ and any small $\delta>0$, let

$$
\begin{aligned}
K_{\theta} & =\max _{x \in B} x \cdot \Theta, \\
r(B, \delta, \theta) & =\left\{y \in B: y \cdot \Theta=K_{\theta}-\delta\right\} .
\end{aligned}
$$

We say that the chord $r(B, \delta, \theta)$ is of height $\delta$, and we use it to define the following inscribed polygon (see also 19] or 23]).

Definition 1. Let $B$ be a convex planar body. Choose any chord of height $\delta$, and name it $c h_{1}$. Move counterclockwise constructing a finite sequence of consecutive chords of height $\delta$ until you reach $c h_{1}$. Then, if necessary, replace the last chord by one consecutive to $c h_{1}$ (hence of height not greater than $\delta$ ). In this way we get a polygon inscribed in $B$, and we denote it by $P_{\delta}^{B}$. Of course $P_{\delta}^{B}$ depends on the choice of $c h_{1}$, and we should write $P_{\delta}^{B}\left(c h_{1}\right)$; however, none of our results depends on $c h_{1}$, and, by a small abuse, we shall always speak about "the" inscribed polygon $P_{\delta}^{B}$. We denote by $M_{\delta}^{B}$ be the number of sides of $P_{\delta}^{B}$.

It has been proved in $[23]$ that $M_{\delta}^{B} \lesssim \delta^{-1 / 2}$. Our first result is the following.

Theorem 2. Let $B$ be a convex planar body and assume $M_{\rho^{-1}}^{B} \lesssim \rho^{\alpha}$ (where $0<$ $\alpha<1 / 2$, the cases $\alpha=0$ and $\alpha=1 / 2$ being covered by (1.3) and (1.2) respectively). Then

$$
\left\|\widehat{\chi}_{B}(\rho \cdot)\right\|_{L^{1}\left(\Sigma_{1}\right)} \lesssim \rho^{\alpha-2} \log \rho .
$$

Moreover, for any $0<\alpha<1 / 2$, there exists a convex planar body $B$ such that $M_{\rho^{-1}}^{B} \lesssim \rho^{\alpha}$ and, for any $\varepsilon>0$,

$$
\limsup _{\rho \rightarrow+\infty} \rho^{-\alpha+2+\varepsilon}\left\|\widehat{\chi}_{B}(\rho \cdot)\right\|_{L^{1}\left(\Sigma_{1}\right)}>0 .
$$

All the proofs will be given in the last section of the paper (§3).

Before going on, we want to discuss the above theorem. The first step in the proof is to show that

$$
\int_{0}^{2 \pi}\left|\widehat{\chi}_{B}(\rho \Theta)\right| d \theta \lesssim \int_{0}^{2 \pi}\left|\widehat{\chi}_{P_{\rho^{-1}}^{B}}(\rho \Theta)\right| d \theta
$$

(see Definition 1). We are therefore reduced to estimating the average decay for a polygon with $\lesssim \rho^{\alpha}$ sides. The second step simply consists in recalling that the implicit constant in (1.3) depends on the number of sides of the polygon $P$, and that after reading the proofs in [7] or [8] one can rewrite (1.3) in the following way:

$$
\int_{0}^{2 \pi}\left|\widehat{\chi}_{P}(\rho \Theta)\right| d \theta \leq c N \rho^{-2} \log \rho,
$$

where $N$ is the number of sides of the polygon $P$, and the constant $c$ is absolute (there is no loss of generality in assuming that the length of the boundary $\partial P$ is $\leq 1)$. Putting $\rho^{\alpha}$ in place of $N$, we then get (1.6).

At this point one should expect to have gotten a poor result using the trivial estimate (1.7). The counterexample in the theorem shows that this is not so. 
1.3. The image of the Gauss map. At every point of $\partial B$ there is a left and a right tangent, therefore a left $(-)$ and a right $(+)$ outward normal. Let $\pi^{ \pm}: \partial B \rightarrow$ $\Sigma_{1}$ be the map sending each point in $\partial B$ to the left/right normal. Also let

$$
\Delta^{B}=\pi^{-}(\partial B) \cup \pi^{+}(\partial B) .
$$

We identify $\Sigma_{1}$ with the interval $[0,2 \pi)$. For every $\theta \in[0,2 \pi)$ we denote by $d\left(\theta, \Delta^{B}\right)$ the distance between $\theta$ and $\Delta^{B}$. For a given small $\delta$, let

$$
\Delta_{\delta}^{B}=\left\{x \in[0,2 \pi): d\left(x, \Delta^{B}\right)<\delta\right\}
$$

be the $\delta$-neighborhood of $\Delta^{B}$.

Theorem 3. Let $0<d<1$. Assume

$$
\left|\Delta_{\delta}^{B}\right| \lesssim \delta^{1-d}
$$

Then

$$
\left\|\widehat{\chi}_{B}(\rho \cdot)\right\|_{L^{1}\left(\Sigma_{1}\right)} \lesssim \rho^{\frac{d}{d+1}-2} .
$$

Moreover, there exists a convex body $B$ satisfying $\left|\Delta_{\delta}^{B}\right| \lesssim \delta^{1-d}$ and such that

$$
\limsup _{\rho \rightarrow+\infty} \rho^{-\frac{d}{d+1}+2+\varepsilon}\left\|\widehat{\chi}_{B}(\rho \cdot)\right\|_{L^{1}\left(\Sigma_{1}\right)}>0
$$

for any $\varepsilon>0$.

The proof will be given in the last section.

Remark 4. Again, the cases $d=0$ and $d=1$ are covered by (1.3) and (1.2) respectively.

Remark 5. We point out that the infimum of the numbers $d$ such that $\left|\Delta_{\delta}^{B}\right| \lesssim \delta^{1-d}$ is just the upper Minkowski dimension of $\Delta^{B}$, that is, the number

$$
d^{*}=\limsup _{\delta \rightarrow 0}\left(\log _{1 / \delta}\left(\left|\Delta_{\delta}^{B}\right| / \delta\right)\right) .
$$

It is therefore possible to restate Theorem 3 in a form such as "If $d>d^{*}$, then (1.11) holds". However, we prefer to keep the original statement in Theorem 3 for the following two reasons. First, the L.H.S. in (1.10) is the quantity that actually arises in the proof. Second, we do not want to confuse naturally different objects, such as the polygons with finitely many sides and certain polygons with infinitely many sides (e.g. with an exponentially decreasing sequence of slopes) which share $d^{*}=0$ with the polygons with finitely many sides. For similar reasons we did not introduce the infimum $\alpha^{*}$ of the $\alpha$ 's in Theorem [2] On the contrary, we shall introduce $\alpha^{*}$ and $d^{*}$ in the following section in order to get a neater comparison.

1.4. Comparing the previous arguments. For any $B$ we denote by $d^{*}$ the Minkowski dimension of $\Delta^{B}$ (see the above remark). We also denote by $\alpha^{*}$ the infimum of the $\alpha$ 's such that $M_{\rho^{-1}}^{B} \leq c_{\alpha} \rho^{\alpha}$. We have the following theorem.

Theorem 6. Let $B$ be a convex planar body. Then

$$
\alpha^{*} \leq \frac{d^{*}}{d^{*}+1}
$$

Moreover, there exists B for which equality holds.

The proof will be given in the last section. 
Remark 7. Theorem [6 exhibits an upper bound for $\alpha^{*}$ in terms of $d^{*}$. A lower bound in terms of $d^{*}$ does not exist in general, since we can construct a family of convex bodies with the same $d^{*}>0$ but $\alpha^{*}$ arbitrarily close to 0 .

The proof will be given in the last section.

The situation is different if we add geometric assumptions on $B$.

Theorem 8. Suppose $B$ is inscribed in a disc (i.e., $B$ is the convex hull of a subset of a circle). Then $\alpha^{*}=d^{*} / 2$.

The proof will be given in the last section.

The circle in the previous statement can be replaced by a closed convex smooth curve with everywhere positive Gaussian curvature.

Remark 9. By appealing to Theorem 2 and Theorem [6 we immediately get the following inequality, which is slightly weaker than the one in Theorem 3

$$
\left\|\widehat{\chi}_{B}(\rho \cdot)\right\|_{L^{1}\left(\Sigma_{1}\right)} \lesssim \rho^{\frac{d}{d+1}-2+\varepsilon} .
$$

1.5. A lower bound for all convex bodies. The main results in this paper deal with "intermediate" cases between polygons and convex bodies having a smooth convex arc in the boundary. These cases turn out be extreme. Indeed, Podkorytov's theorem is a uniform (with respect to the choice of $B$ ) upper bound, while the following theorem gives a uniform lower bound for the $L^{1}$ average decay of the Fourier transform.

Theorem 10. Let $B$ be a convex body in $\mathbb{R}^{2}$. Then

$$
\limsup _{\rho \rightarrow+\infty} \rho^{2} \log ^{-1} \rho\left\|\widehat{\chi}_{B}(\rho \cdot)\right\|_{L^{1}\left(\Sigma_{1}\right)}>0 .
$$

The proof will be given in the last section.

\section{Applichtions}

2.1. Lattice points. Let $B$ be a planar convex body, and let $\sigma \in S O(2)$ and $t \in \mathbb{T}^{2}$. We consider the discrepancy

$$
D_{\rho}(B)=\operatorname{card}\left(\rho B \cap \mathbb{Z}^{2}\right)-\rho^{2}|B|,
$$

where $|\cdot|$ denotes the area. The results in the previous section and some arguments in [20], 25], [7], and [8] allow us to obtain several upper and lower bounds for averages of the discrepancy (2.1) over rotations or rotations and translations. As a first example, it has been proved in [15], 25], and [7] that, for a polygon $P,(1.3)$ implies

$$
\int_{S O(2)}\left|D_{\rho}\left(\sigma^{-1}(P)\right)\right| d \sigma \lesssim \log ^{2} \rho .
$$

As another example, one can use (1.2) to show that for any convex planar body $B$,

$$
\left\{\int_{\mathbb{T}^{2}} \int_{S O(2)}\left|D_{\rho}\left(\sigma^{-1}(P)-t\right)\right|^{2} d \sigma d t\right\}^{1 / 2} \lesssim \rho^{1 / 2} .
$$

(See e.g. [15] or [8].) Note that (2.2) is false without the integration in $t$, as the case of a disc and Hardy's $\Omega$-result (see [16]) show.

Again we focus on the case $p=1$, and we have the following result, which follows easily from Theorem 2 and some known arguments (see e.g. [15], [25] or [7]). 
Theorem 11. Let $B$ be a planar convex body such that $M_{\rho^{-1}}^{B} \lesssim \rho^{\alpha}(0<\alpha<1 / 2)$. Then

$$
\int_{\mathbb{T}^{2}} \int_{S O(2)}\left|D_{\rho}\left(\sigma^{-1}(B)-t\right)\right| d \sigma d t \lesssim \rho^{\frac{2 \alpha}{2 \alpha+1}} \log \rho .
$$

Moreover, for every such $\alpha$ there exists a body B satisfying

$$
\limsup _{\rho \rightarrow+\infty} \rho^{-\alpha+\varepsilon} \int_{\mathbb{T}^{2}} \int_{S O(2)}\left|D_{\rho}\left(\sigma^{-1}(B)-t\right)\right| d \sigma d t>0,
$$

for any $\varepsilon>0$.

The proof will be given in the last section.

Remark 12. The cases $\alpha=0$ and $\alpha=1 / 2$ are known; see e.g. [7] and [8] respectively.

2.2. Irregularities of distribution. Suppose $\mathcal{P}=\left\{z_{j}\right\}_{j=1}^{N}$ is a distribution of $N$ points in the unit square $U=[0,1]^{2}$ treated as the torus $\mathbb{T}^{2}$. Let $B$ be a convex body in $U$ with diameter smaller than 1 . Assume $\varepsilon \leq 1, \sigma \in S O(2)$, and $t \in \mathbb{T}^{2}$. The study of the discrepancy

$$
D(\mathcal{P}, \varepsilon, \sigma, t)=\sum_{j=1}^{N} \chi_{\varepsilon \sigma^{-1} B-t}\left(z_{j}\right)-N \varepsilon^{2}|B|
$$

has a long history (see e.g. the references in [2] and [17, ch. 6]). A typical result is the following theorem, due to Beck [1] and Montgomery [17, ch. 6] (see also [7]).

Theorem 13. Let $B$ be a convex body in $U=[0,1]^{2}$ with diameter smaller than 1 . Then, for every distribution $\mathcal{P}=\left\{z_{j}\right\}_{j=1}^{N}$ in $U$,

$$
\left\{\int_{0}^{1} \int_{S O(2)} \int_{\mathbb{T}^{2}}|D(\mathcal{P}, \varepsilon, \sigma, t)|^{2} d t d \sigma d \varepsilon\right\}^{1 / 2} \gtrsim N^{1 / 4} .
$$

The above result is sharp, since Beck and Chen 3 proved the following upper bound.

Theorem 14. Let $B$ be a convex body in $U=[0,1]^{2}$ with diameter smaller than 1. Then for every positive integer $N$ there exists a distribution $\mathcal{P}$ of $N$ points such that

$$
\left\{\int_{0}^{1} \int_{S O(2)} \int_{\mathbb{T}^{2}}|D(\mathcal{P}, \varepsilon, \sigma, t)|^{2} d t d \sigma d \varepsilon\right\}^{1 / 2} \lesssim N^{1 / 4} .
$$

The above upper bound can be improved after replacing the $L^{2}$ norm with the $L^{1}$ norm. Indeed, Beck and Chen [4] proved the following result.

Theorem 15. Let $P$ be a convex polygon in $U=[0,1]^{2}$ with diameter smaller than 1. Then for every positive integer $N$ there exists a distribution $\mathcal{P}$ of $N$ points such that

$$
\int_{0}^{1} \int_{S O(2)} \int_{\mathbb{T}^{2}}|D(\mathcal{P}, \varepsilon, \sigma, t)| d t d \sigma d \varepsilon \lesssim \log ^{2} N
$$


The next result follows easily from Theorem 11, [7] and [8]. The case $\alpha=0$ provides a different proof of (2.5). In the same way one can get a different proof of the $L^{2}$ result in (2.4) too. We point out that appealing to lattice point results does not work for $L^{p}$ norms when $p>2$ and the body is a polygon (see [11]).

Theorem 16. Let $B$ be a convex body in $U=[0,1]^{2}$ with diameter smaller than 1 and such that $M_{\rho^{-1}}^{B} \lesssim \rho^{\alpha}$. Then for every positive integer $N$ there exists a distribution $\mathcal{P}$ of $N$ points satisfying

$$
\int_{\mathbb{T}^{2}} \int_{S O(2)}|D(\mathcal{P}, \sigma, t)| d \sigma d t \lesssim \begin{cases}\log ^{2} N & \text { when } \alpha=0, \\ N^{\frac{\alpha}{1+2 \alpha}} \log N & \text { when } 0<\alpha<1 / 2, \\ N^{1 / 4} & \text { when } \alpha=1 / 2,\end{cases}
$$

where $D(\mathcal{P}, \sigma, t)=D(\mathcal{P}, 1, \sigma, t)$.

The proof will be given in the last section.

\section{Proofs}

The following known result (see e.g. [10], 19], 8]) will be used throughout the paper.

Lemma 17. Let $B$ be a convex body in $\mathbb{R}^{2}$. Using the notation in 1.5 , we have

$$
\left|\widehat{\chi}_{B}(\rho \Theta)\right| \lesssim \rho^{-1}\left[\left|r\left(B, \rho^{-1}, \theta\right)\right|+\left|r\left(B, \rho^{-1}, \theta+\pi\right)\right|\right],
$$

where $|\cdot|$ denotes the length of the chord.

We define

$$
\widetilde{d}\left(\theta, \Delta^{B}\right)=\min \left(d\left(\theta, \Delta^{B}\right), d\left(\theta+\pi, \Delta^{B}\right)\right),
$$

and we deduce the following lemma.

Lemma 18. For every $\theta \notin \Delta^{B}$ we have

$$
\left|\widehat{\chi}_{B}(\rho \Theta)\right| \lesssim \frac{1}{\rho^{2} \widetilde{d}\left(\theta, \Delta^{B}\right)}
$$

Proof. Let $\theta \notin \Delta^{B}$ (say $\theta=-\pi / 2$ ). Assume that $\partial B$ passes through the origin and that $B$ lies in the upper half-plane. It follows that in a neighborhood of the origin $\partial B$ is the graph of a nonnegative convex function, say $y=\varphi(x)$, satisfying $\varphi(0)=0$ and $\varphi^{\prime}(0-)<0<\varphi^{\prime}(0+)$, where $\varphi^{\prime}(0-)$ and $\varphi^{\prime}(0+)$ denote the left and the right derivative at the origin respectively. Let

$$
E=\left\{(x, y) \in \mathbb{R}^{2}: y>\varphi^{\prime}(0-) x \text { and } y>\varphi^{\prime}(0+) x\right\} .
$$

By convexity, $B \subset E$, and therefore

$$
\left|r\left(B, \rho^{-1}, \theta\right)\right| \leqslant \frac{1}{\rho \varphi^{\prime}(0+)}+\frac{1}{\rho\left|\varphi^{\prime}(0-)\right|} \leqslant \frac{2}{\rho \min \left(\varphi^{\prime}(0+),\left|\varphi^{\prime}(0-)\right|\right)} .
$$

To complete the proof it is enough to observe that

$$
\min \left(\varphi^{\prime}(0+),\left|\varphi^{\prime}(0-)\right|\right) \approx d\left(\theta, \Delta^{B}\right)
$$

and to apply the previous lemma.

The following lemmas will be needed in the proof of Theorem 2 
Lemma 19. Let $R \geq 1$ and $0<\beta<\pi / 4$. Assume $R \beta<1 / 2$. Denote by $C=C(\beta, R)$ the convex hull of the set

$$
\{R \exp (i \theta):-\beta \leq \theta \leq \beta\} \cup\{P\},
$$

where the point $P$ has distance 1 from the points $R^{ \pm i \beta}$ and satisfies $|P| \leq R$. Then there exist positive constants $c_{1}$ and $c_{2}$ such that if $R \rho \beta^{2} \geqslant c_{1}$ then we have

$$
\left|\widehat{\chi}_{C}(\rho \Theta)\right| \geqslant c_{2} R^{1 / 2} \rho^{-3 / 2}
$$

for every $|\theta| \leq \beta / 2$.

Proof. Integrating by parts, we are reduced to estimating

$$
\rho^{-1} \int_{\partial C} n(x) \cdot \Theta \exp (2 \pi i \rho \Theta \cdot x) d x .
$$

The boundary $\partial C$ consists of two segments and an arc. In order to control the latter we reduce to the oscillatory integral

$$
\left|\int_{-R \beta}^{R \beta} \exp \left(i \rho \frac{t^{2}}{R}\right) d t\right|=\left|R \beta \int_{-1}^{1} \exp \left(i \rho R \beta^{2} u\right) d u\right| \geqslant c R^{1 / 2} \rho^{-1 / 2}
$$

for $\rho R \beta^{2}$ large enough. The two segments have length 1 , and their contribution in (3.1) is $O\left(\rho^{-2}\right)$.

Lemma 20. Let $R>1$ and $0<\beta<\pi / 4$. Assume $R \beta<\frac{1}{2}$. For any $N \geq 1$, let $B=B(\beta, R, N)$ be the convex hull of the set

$$
\{R \exp (2 \pi i k \beta / N), k=-N, \ldots, N\} \cup\{P\},
$$

where, as before, the point $P$ has distance 1 from the points $R e^{ \pm i \beta}$ and satisfies $|P| \leq R$. Then there exist absolute constants $c_{1}, c_{2}$, and $c_{3}$ such that whenever $\rho \geq 2$ and

$$
\frac{c_{1}}{\beta^{2}} \leq R \rho \leq \frac{c_{2}}{\beta^{2}} \frac{N^{2}}{\log ^{2} N},
$$

we have, for any $-\beta / 2 \leq \theta \leq \beta / 2$,

$$
\left|\widehat{\chi}_{B}(\rho \Theta)\right| \geq c_{3} R^{1 / 2} \rho^{-3 / 2} .
$$

Proof. Let $C=C(\beta, R)$ be as in Lemma 19, By (3.2) and Lemma 19 we have

$$
\left|\widehat{\chi}_{C}(\rho \Theta)\right| \geq c R^{1 / 2} \rho^{-3 / 2}
$$

when $-\beta / 2 \leq \theta \leq \beta / 2$.

We now study the Fourier transform $\widehat{\chi}_{C \backslash B}$. We claim that

$$
\left|\widehat{\chi}_{C \backslash B}(\rho \Theta)\right| \leq c \beta \rho^{-1} \frac{\log N}{N} R
$$

uniformly in $\theta$. Indeed, $C \backslash B$ is the union of $2 N$ "lunes" $\ell_{1}, \ldots, \ell_{2 N}$ (each lune is a convex set bounded by a segment in $B$ and by a portion of the arc in $C$, see Figure 1) and, for any $\theta$,

$$
\widehat{\chi}_{C \backslash B}(\rho \Theta)=\widehat{f}(\rho),
$$


where $f=f_{\theta}$ is defined by

$$
\begin{aligned}
f(s) & =\left|C \backslash B \cap\left\{\xi \in \mathbb{R}^{2}: \xi \cdot \Theta=s\right\}\right| \\
& =\sum_{k=1}^{2 N}\left|\ell_{k} \cap\left\{\xi \in \mathbb{R}^{2}: \xi \cdot \Theta=s\right\}\right| \\
& =\sum_{k=1}^{2 N} f_{k}(s) .
\end{aligned}
$$

Note that, for any given $s$, the above sum contains at most two terms. It is enough to consider one of them, i.e., we assume $0 \leq \theta \leq \pi$. Moreover, we are reduced to studying the case $0 \leq \theta<\beta / N$, the other cases being similar. In order to bound $\widehat{f}(\rho)$, we estimate the total variation $V_{f}$ of the function $f(s)$, which is the length of the vertical segment in the $k$ th lune. Now observe that

$$
V_{f_{k}} \leq c \beta N^{-1} k^{-1} R
$$

whenever $k \geq 1$ (see Figure 1).

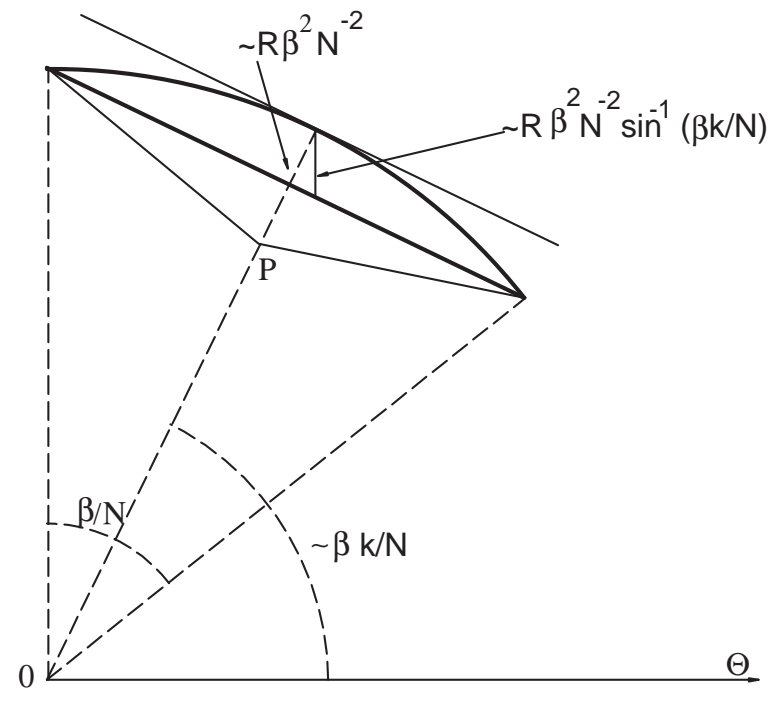

FiguRE 1.

Summing on $k$ (there are $N$ terms when $\theta=0$ and $N+1$ terms when $0<\theta<$ $\beta / N)$, we get (3.3).

Finally, for suitable choices of $c_{1}$ and $c_{2}$ in (3.2) we get

$$
\begin{aligned}
\left|\widehat{\chi}_{B}(\rho \Theta)\right| & \geq\left|\widehat{\chi}_{C}(\rho \Theta)\right|-\left|\widehat{\chi}_{B \backslash C}(\rho \Theta)\right| \\
& \geq c_{3} R^{1 / 2} \rho^{-3 / 2}-c_{4} \beta \rho^{-1} \frac{\log N}{N} R \\
& \geq c_{5} \rho^{-3 / 2} R^{1 / 2} .
\end{aligned}
$$

Proof of Theorem [. We start with the upper bounds in (1.6). Let $P_{\rho^{-1}}^{B}$ be as in Definition 11 Let $\widetilde{P}_{\rho^{-1}}^{B}$ be the smallest polygon having sides parallel to those of 
$P_{\rho}^{B}$ and containing $B$. It is not difficult to see that for $\rho$ sufficiently large,

$$
\left|r\left(B, \rho^{-1}, \theta\right)\right| \lesssim\left|r\left(\widetilde{P}_{\rho}, c \rho^{-1}, \theta\right)\right|,
$$

where again the implicit constant depends only on $B$. By Lemma 17 we have

$$
\begin{aligned}
\left|\widehat{\chi}_{B}(\rho \Theta)\right| & \lesssim \rho^{-1}\left|r\left(B, \rho^{-1}, \theta\right)\right| \\
& \lesssim \rho^{-1}\left|r\left(\widetilde{P}_{\rho^{-1}}, c \rho^{-1}, \theta\right)\right| .
\end{aligned}
$$

Hence, by the proof of (1.7) in [7] or [8],

$$
\rho^{-1} \int_{0}^{2 \pi}\left|r\left(\widetilde{P}_{\rho^{-1}}, c \rho^{-1}, \theta\right)\right| d \theta \leq c M_{\rho^{-1}}^{B} \rho^{-2} \log (\rho) \leq c \rho^{-2+\alpha} \log (\rho),
$$

thereby proving (1.6).

We now show that (1.6) is essentially sharp. Let $B=B(\beta, R, N)$ be as in Lemma 20 and consider the sets $B_{h}=B\left(\beta_{h}, R_{h}, N_{h}\right), h=1,2,3, \ldots$, where, for any small $\varepsilon>0$,

$$
R_{h}=2^{(1-2 \alpha) h}, \quad \beta_{h}=2^{h(2 \alpha-1-\varepsilon)}, \quad N_{h}=2^{h \alpha} .
$$

We denote by $\gamma_{h}$ the union of the $N_{h}$ sides and by $\zeta_{h}$ the arc where they are inscribed. Observe that

$$
\sum_{h=n_{0}}^{+\infty} \beta_{h} R_{h}<\pi / 4
$$

for a suitable $n_{0}$.

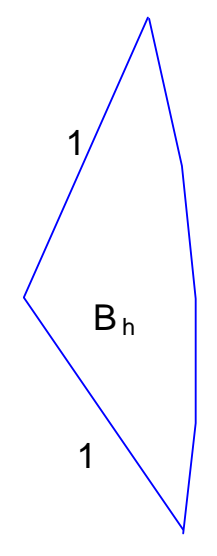

FIGURE 2.

We recall that each $B_{h}$ has the shape in Figure 2, i.e., it is a convex polygon consisting of two sides of length 1 and of $N_{h}$ sides coming from a regular polygon of large radius $R_{h}$. Let $E_{h}$ be the rotated and translated copy of every $B_{h}$, so that, moving counterclockwise, $E_{n_{0}}=B_{n_{0}}$ and two consecutive $E_{h}$ 's have disjoint 
interior and share a side (of length 1), while the union of the $\operatorname{arcs} \zeta_{h}$ is a convex piecewise smooth curve. We write

$$
B=\left(\bigcup_{j=n_{0}}^{h-1} E_{j}\right) \cup E_{h} \cup\left(\bigcup_{j=h+1}^{\infty} E_{j}\right)=\widetilde{E}_{h} \cup E_{h} \cup E_{h}^{\#} .
$$

By the condition (3.4),$B$ is a convex set. Now let $\rho_{h}=2^{h}$. Let $p_{h}=\sum_{j=n_{0}}^{h} \beta_{j}$. Since (3.2) is satisfied, Lemma 20 implies

$$
\left|\widehat{\chi}_{E_{h}}\left(\rho_{h} \Theta\right)\right| \geq c R_{h}^{1 / 2} \rho_{h}^{-3 / 2}=c 2^{-h(\alpha+1)}
$$

for

$$
p_{h}+\frac{1}{3} \beta_{h}<\theta<p_{h}+\frac{2}{3} \beta_{h} .
$$

We then estimate the contribution of the convex sets $\widetilde{E}_{h}$ and $E_{h}^{\#}$, using Lemma 18 Indeed, since $\theta$ satisfies (3.6), we obtain, for any $h$,

$$
\left|\widehat{\chi}_{\widetilde{E}_{h}}\left(\rho_{h} \Theta\right)\right|+\left|\widehat{\chi}_{E_{h}^{\#}}\left(\rho_{h} \Theta\right)\right| \leq c \beta_{h}^{-1} \rho_{h}^{-2} .
$$

We then have

$$
\begin{aligned}
\int_{0}^{2 \pi}\left|\widehat{\chi}_{B}\left(\rho_{h} \Theta\right)\right| d \theta & \geq \int_{p_{h}+\frac{1}{3} \beta_{h}}^{p_{h}+\frac{2}{3} \beta_{h}}\left|\widehat{\chi}_{B}\left(\rho_{h} \Theta\right)\right| d \theta \\
& \geq\left|c_{1} \beta_{h} R_{h}^{1 / 2} \rho_{h}^{-3 / 2}-c_{2} \rho_{h}^{-2}\right| \\
& \geq\left|c_{1} 2^{h(\alpha-\varepsilon-2)}-c_{2} 2^{-2 h}\right| \\
& \geq c_{3} \rho_{h}^{-2+\alpha-\varepsilon} .
\end{aligned}
$$

To complete the proof we estimate $M_{\rho^{-1}}^{B}$. Given $\rho \geq 2$, let $H$ satisfy $2^{H} \leq \rho<2^{H+1}$. Here we split

$$
B=\left(\bigcup_{j=n_{0}}^{H} E_{j}\right) \cup\left(\bigcup_{j=H+1}^{+\infty} E_{j}\right)=B_{a} \cup B_{b} .
$$

Observe that the first term is a polygon with $\sum_{j=n_{0}}^{H} N_{j} \lesssim 2^{H \alpha}$ sides. Now consider that for any convex polygon $Q$ and any $\delta$, the number $M_{\delta}^{Q}$ cannot exceed the number of sides of $Q$. Therefore the contribution of $B_{a}$ to $M_{\rho^{-1}}^{B}$ is $\lesssim 2^{H \alpha}=\rho^{\alpha}$. As for $B_{b}$, we note that the length of $\bigcup_{j=H+1}^{+\infty} \zeta_{j}$ is comparable to the length of $\zeta_{H}$, while the chords of height $\rho^{-1}$ are longer, since $\bigcup_{j=H+1}^{+\infty} \zeta_{j}$ comes from flatter arcs. Therefore there are fewer chords than for $\zeta_{H}$. We have therefore proved that $M_{\rho^{-1}}^{B} \lesssim \rho^{\alpha}$.

Proof of Theorem 3 . Let $\Omega_{\rho}=\Delta_{\rho^{-1 /(d+1)}}^{B}$. In order to estimate

$$
I(\rho)=\int_{0}^{2 \pi}\left|\widehat{\chi}_{B}(\rho \Theta)\right| d \theta
$$

we write

$$
I(\rho)=\int_{\Omega_{\rho}}\left|\widehat{\chi}_{B}(\rho \Theta)\right| d \theta+\int_{[0,2 \pi] \backslash \Omega_{\rho}}\left|\widehat{\chi}_{B}(\rho \Theta)\right| d \theta=I_{1}+I_{2} .
$$


To estimate $I_{1}$ we use the Cauchy-Schwarz inequality, the fact that $\left|\Delta_{\delta}^{B}\right| \lesssim \delta^{1-d}$, and (1.2):

$$
\begin{aligned}
I_{1} & \leq\left|\Omega_{\rho}\right|^{1 / 2}\left\{\int_{0}^{2 \pi}\left|\widehat{\chi}_{B}(\rho \Theta)\right|^{2} d \theta\right\}^{1 / 2} \\
& \lesssim \rho^{(d-1) /(2 d+2)} \rho^{-3 / 2} \\
& =c \rho^{-2+\frac{d}{d+1}} .
\end{aligned}
$$

In order to estimate $I_{2}$ we use Lemma 18 .

$$
\begin{aligned}
I_{2} & \lesssim \sum_{k=0}^{(d+1)^{-1} \log \rho} \int_{\Delta_{2^{-}-}^{B} \backslash \Delta_{2^{-k-1}}} \frac{c}{\rho^{2} \widetilde{d}\left(\theta, \Delta^{B}\right)} d \theta \\
& \lesssim \rho^{-2} \sum_{k=0}^{(d+1)^{-1} \log \rho} 2^{k}\left|\Delta_{2^{-k}}^{B}\right| \\
& \lesssim \rho^{-2} \sum_{k=0}^{(d+1)^{-1} \log \rho} 2^{k} 2^{-k(1-d)} \\
& \lesssim \rho^{-2} \sum_{k=0}^{(d+1)^{-1} \log \rho} 2^{k d} \\
& =c \rho^{-2+\frac{d}{d+1}} .
\end{aligned}
$$

In order to give a counterexample we use the body $B$ constructed in the proof of Theorem 22 Again we consider the sets $B_{h}=B\left(\beta_{h}, R_{h}, N_{h}\right), h=1,2, \ldots$, where now

$$
R_{h}=2^{h \frac{1-d}{1+d}}, \quad \beta_{h}=2^{h\left(\frac{d-1}{d+1}-\varepsilon\right)}, \quad N_{h}=2^{h \frac{d}{d+1}},
$$

while $\rho_{h}=2^{h}$. Arguing as in the proof of the previous theorem, we get, for every $h$,

$$
\rho_{h}^{2-\frac{d}{1+d}+\varepsilon} \int_{0}^{2 \pi}\left|\widehat{\chi}_{B}\left(\rho_{h} \Theta\right)\right| d \theta \geq c .
$$

To complete the proof it is enough to show that $\left|\Delta_{\delta}^{B}\right| \lesssim \delta^{1-d}$. We identify $\Delta_{\delta}^{B}$ with a subset of $[0, \pi / 2]$, and we observe that

$$
\Delta_{\delta}^{B} \cap\left[\sum_{j \leq H-1} \beta_{j}, \sum_{j \leq H} \beta_{j}\right]
$$

consists of $N_{H}$ points at distance $\beta_{H} / N_{H}$. Given $\delta>0$, we choose $H$ so that

$$
\frac{\beta_{H}}{N_{H}} \leq \delta<\frac{\beta_{H-1}}{N_{H-1}}
$$

Hence

$$
\beta_{H} \leq\left(\frac{\beta_{H}}{N_{H}}\right)^{1-d} \approx \delta^{1-d} .
$$

We now split $B=B_{a} \cup B_{b}$ as in (3.7). The contribution of $B_{a}$ to $\left|\Delta_{\delta}^{B}\right|$ is

$$
\delta \sum_{j \leq H} N_{j} \approx \delta N_{H} \approx \beta_{H} \lesssim \delta^{1-d}
$$


while the contribution of $B_{b}$ to $\left|\Delta_{\delta}^{B}\right|$ is bounded by

$$
\sum_{j>H} \beta_{j} \lesssim \beta_{H} \lesssim \delta^{1-d}
$$

The next proof follows an argument in [23].

Proof of Theorem 6. Let $c h_{j}$ be a side of $P_{\rho^{-1}}^{B}$ having endpoints $x_{j}$ and $y_{j}$. Assume that, moving counterclockwise along the boundary of $B$, the point $x_{j}$ comes before $y_{j}$. Denote by $\varphi_{j}$ the direction of the right normal in $x_{j}$ and by $\psi_{j}$ the direction of the left normal in $y_{j}$. First observe that

$$
\left|c h_{j}\right|\left|\varphi_{j}-\psi_{j}\right| \gtrsim \rho^{-1} \text {. }
$$

((3.8) follows by convexity when $\left|\varphi_{j}-\psi_{j}\right| \geq \pi / 4$ and by a trigonometric computation when $\left|\varphi_{j}-\psi_{j}\right|<\pi / 4$.) Let $\alpha>\alpha^{*}$. Summing up and applying the Hölder inequality, we get

$$
\begin{aligned}
\rho^{-\alpha} M_{\rho^{-1}}^{B} & \lesssim \sum_{j}\left|c h_{j}\right|^{\alpha}\left|\varphi_{j}-\psi_{j}\right|^{\alpha} \\
& \leq\left\{\sum_{j}\left|c h_{j}\right|\right\}^{\alpha}\left\{\sum_{j}\left|\varphi_{j}-\psi_{j}\right|^{\frac{\alpha}{1-\alpha}}\right\}^{1-\alpha} \\
& \leq|\partial B|^{\alpha}\left(\sum_{j}\left|\varphi_{j}-\psi_{j}\right|^{\frac{\alpha}{1-\alpha}}\right)^{1-\alpha}
\end{aligned}
$$

where the sum is on the $M_{\rho^{-1}}^{B}$ sides of the polygon $P_{\rho^{-1}}$. It remains to show that $\sum_{j}\left|\varphi_{j}-\psi_{j}\right|^{\frac{\alpha}{1-\alpha}}$ is bounded by a constant independent of $P_{\rho^{-1}}$. Let

$$
Z_{k}=\left\{j: 2^{-k} \pi<\left|\varphi_{j}-\psi_{j}\right| \leq 2^{1-k} \pi\right\} .
$$

Now observe that if $j \in Z_{k}$, then the interval $\left(\varphi_{j}, \psi_{j}\right) \subseteq \Delta_{2^{-k} \pi}^{B}$. Now choose $d$ such that $d^{*}<d<\frac{\alpha}{1-\alpha}$. Then

$$
2^{-k} \pi \operatorname{card}\left(Z_{k}\right) \leq\left|\Delta_{2^{-k} \pi}^{B}\right| \lesssim 2^{-k(1-d)},
$$

so that $\operatorname{card}\left(Z_{k}\right) \lesssim 2^{k d}$, and therefore

$$
\begin{aligned}
\sum_{j}\left|\varphi_{j}-\psi_{j}\right|^{\frac{\alpha}{1-\alpha}} & \leq \sum_{k=0}^{+\infty} \sum_{j \in Z_{k}}\left|\varphi_{j}-\psi_{j}\right|^{\frac{\alpha}{1-\alpha}} \\
& \lesssim \sum_{k=0}^{+\infty} 2^{k d} 2^{-k \frac{\alpha}{1-\alpha}} \\
& =\sum_{k=0}^{+\infty} 2^{-k\left(\frac{\alpha}{1-\alpha}-d\right)} \\
& <+\infty .
\end{aligned}
$$

The sharpness of the inequality $\alpha^{*} \leq \frac{d^{*}}{d^{*}+1}$ follows from the common counterexample in the proof of Theorem 2 and Theorem 3 , 
Proof of Remark 7 . Let $\gamma>1$ and $\beta>0$. For $n \geq 1$, let $x_{n}=n^{-\beta}$ and $y_{n}=n^{-\beta \gamma}$. Let $B$ denote the convex hull of the infinite points $\left(x_{n}, y_{n}\right)$. We claim that the polygon $P_{\rho^{-1}}$ associated to $B$ satisfies

$$
M_{\rho^{-1}}^{B} \lesssim \rho^{\frac{1}{\gamma \beta}}
$$

(hence $\alpha^{*} \leq 1 / \gamma \beta$ ). Indeed, choose

$$
c h_{1}=B \cap\left\{(x, y) \in \mathbb{R}^{2}: y=\frac{1}{\rho}\right\}
$$

as the first side of $P_{\rho^{-1}}$. The number of sides of $B$ located on the right of $c h_{1}$ is $\approx \rho^{1 / \gamma \beta}$, and the claim follows since for any polygon $D$ with finitely many sides and any $\rho$ we have $M_{\rho^{-1}}^{D} \leq \#$ (sides of $\left.D\right)$. On the other hand, one checks that $B$ satisfies

$$
\left|\Delta_{\delta}^{B}\right| \lesssim \delta^{1-\frac{1}{\beta(\gamma-1)+1}}
$$

and the exponent is the best possible (i.e., $\left.d^{*}=1 /(\beta(\gamma-1)+1)\right)$.

If we now choose $\gamma=1+1 / \beta$, we get $d^{*}=1 / 2$ and $\alpha^{*}$ arbitrarily small (since $\beta$ can be large).

Proof of Theorem 8 . We show that $\alpha^{*}=d^{*} / 2$ whenever $B$ is inscribed in a disc, namely when $B$ is the convex hull of a subset of a circle.

Let $P_{\rho^{-1}}^{B}$ be as in Definition 1 and assume $\alpha>\alpha^{*}$, hence $M_{\rho^{-1}}^{B} \lesssim \rho^{\alpha}$. Let $x_{1}, x_{2}, \ldots$ be the vertices of $P_{\rho^{-1}}^{B}$. See Figure 3 .

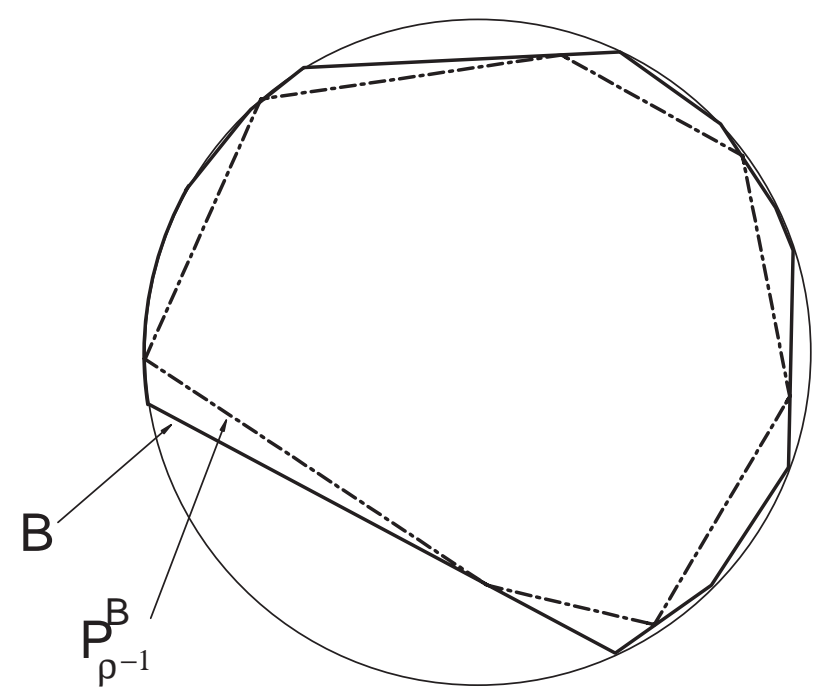

FiguRE 3.

Let $B_{1}, B_{2}, \ldots$ be discs of radius $\rho^{-1 / 2}$ centered at the above vertices. Since $B$ is the convex hull of a subset of a given circle $C$, there exists a constant $c$ such that, for any $j$, we are in at least one of the following two cases: 
either

i) $c B_{j} \cup c B_{j+1}$ contains the arc in $\partial B$ connecting $x_{j}$ and $x_{j+1}$,

or

ii) the part of $\partial B$ connecting $x_{j}$ and $x_{j+1}$ and not contained in $c B_{j} \cup c B_{j+1}$ is a segment.

Indeed, assume that $i$ ) and $i i$ ) fail. Then the arc in $\partial B$ connecting $x_{j}$ and $x_{j+1}$ must touch the unit circle $C$ outside of the $\operatorname{discs} c B_{j}$ or $c B_{j+1}$, at a point having distance $\approx \rho^{-1}$ from the side of $P_{\rho^{-1}}^{B}$ connecting $x_{j}$ and $x_{j+1}$. Now observe that this latter can be extended to a chord of $C$ at distance $\approx \rho^{-1}$ from $\partial C$. Then, for a suitable $c$, the discs $c B_{j}$ and $c B_{j+1}$ cannot be distinct.

The above implies that, for $\alpha>\alpha^{*}$,

$$
\Delta_{\rho^{-1 / 2}}^{B} \subseteq c_{1} \pi^{ \pm}\left(\partial B \cap\left(\bigcup_{j=1}^{c \rho^{\alpha}} c B_{j}\right)\right),
$$

and therefore

$$
\left|\Delta_{\rho^{-1 / 2}}^{B}\right| \lesssim \sum_{j=1}^{c \rho^{\alpha}} \rho^{-1 / 2} \approx \rho^{\alpha-1 / 2}=\left(\rho^{-1 / 2}\right)^{1-2 \alpha} ;
$$

hence, in this case, $d^{*} \leq 2 \alpha^{*}$.

We now prove that $\alpha^{*} \leq d^{*} / 2$. Let $\bar{\alpha}<\alpha^{*}$. Then there exists a sequence $\rho_{k} \rightarrow+\infty$ such that $M_{\rho_{k}^{-1}}^{B} \gtrsim \rho_{k}^{\bar{\alpha}}$. We claim that there exist $\approx \rho_{k}^{\bar{\alpha}}$ points in $\Delta^{B}$ that are $\approx \rho_{k}^{-1 / 2}$ separated. Postponing for a moment the proof of the claim, we conclude that

$$
\left|\Delta_{\rho_{k}^{-1 / 2}}^{B}\right| \gtrsim \rho_{k}^{\bar{\alpha}-1 / 2}=\left(\rho_{k}^{-1 / 2}\right)^{1-2 \bar{\alpha}}
$$

which implies that the Minkowski dimension $d^{*}$ of $\Delta^{B}$ cannot be smaller than $2 \bar{\alpha}$, and therefore $d^{*} \geq 2 \alpha^{*}$.

Proof of the claim. Let $c h_{j}, \varphi_{j}$ and $\psi_{j}$ be as in the proof of Theorem $[6$ and define

$$
\begin{aligned}
& S_{a}=\left\{j:\left|\varphi_{j}-\psi_{j}\right|>\rho_{k}^{-1 / 2}\right\}, \\
& S_{b}=\left\{j:\left|\varphi_{j}-\psi_{j}\right| \leq \rho_{k}^{-1 / 2}\right\} .
\end{aligned}
$$

It is enough to prove that whenever $j \in S_{b}$ we have $\left|\varphi_{j}-\psi_{j}\right| \gtrsim c \rho_{k}^{-1 / 2}$. Since $B$ is inscribed in a (unit) circle, a simple geometric argument shows that if $\left|\varphi_{j}-\psi_{j}\right| \leq$ $\rho_{k}^{-1 / 2}$, then the chord $c h_{j}$ (which is a chord of $B$ of height $\rho_{k}^{-1}$ ) can be continued to a chord of the circle of height $\approx \rho_{k}^{-1}$ and therefore of length $\approx \rho_{k}^{-1 / 2}$. It follows that $\left|c h_{j}\right| \lesssim \rho_{k}^{-1 / 2}$, and (3.8) yields $\left|\varphi_{j}-\psi_{j}\right| \gtrsim c \rho_{k}^{-1 / 2}$ for any $j=1, \ldots, c \rho^{\alpha}$.

The following lemma will be needed in the proof of Theorem 10. The proof depends on an easy modification of an argument in [27.

Lemma 21. Let $B$ be a convex planar body containing a large disc of radius $r$. Let $g$ be a smooth nonnegative function supported in the set $\{t+v\}_{t \in B,|v| \leq 1}$ such that $g(t)=1$ when $t \in B$ and $\operatorname{dist}(t, \partial B) \geq 1$. Then there exists a constant $c$, independent of $r$, such that

$$
\|\widehat{g}\|_{L^{1}\left(\mathbb{R}^{2}\right)} \geq c \log ^{2} r
$$


Proof. We first need the following known inequality (see e.g. [24] or [13]). Let $h \in L^{1}(\mathbb{R})$ satisfy $\widehat{h} \in L^{1}(\mathbb{R})$ and $\widehat{h}(u)=0$ for $u \leq 0$. Then

$$
\int_{-\infty}^{+\infty}|h(x)| d x \geq c \int_{1}^{+\infty} \frac{1}{u}|\widehat{h}(u)| d u .
$$

A quick proof of (3.9) follows. Because of [12, p. 584] we can assume $\widehat{h}(u) \geq 0$. We then consider the odd real function $s$ defined by $s(x)=-i(1-x)_{+}$for $x>0$, the Fourier transform of which is $\widehat{s}(u)=(2 \pi u-\sin 2 \pi u) / 2 \pi^{2} u^{2}$. Then

$$
\begin{aligned}
\int_{-\infty}^{+\infty}|h(x)| d x & \geq\left|\int_{-\infty}^{+\infty} h(x) s(x) d x\right| \\
& =\left|\int_{-\infty}^{+\infty} \widehat{h}(u) \widehat{s}(u) d u\right| \\
& \geq c \int_{1}^{+\infty} \frac{\widehat{h}(u)}{u} d u .
\end{aligned}
$$

Observe that, through a translation, (3.9) implies the following fact. Suppose $\widehat{h}(u)=1$ for $u$ in an interval of length $r$, say $[q, q+r]$, and that, moreover, $\widehat{h}(u)=0$ for $u \leq q-1$. Then

$$
\int_{-\infty}^{+\infty}|h(x)| d x \geq c \log r
$$

To prove the lemma we may suppose that $B$ lies in the half-plane $\{(x, y): x \geq 1\}$ as in Figure 4.

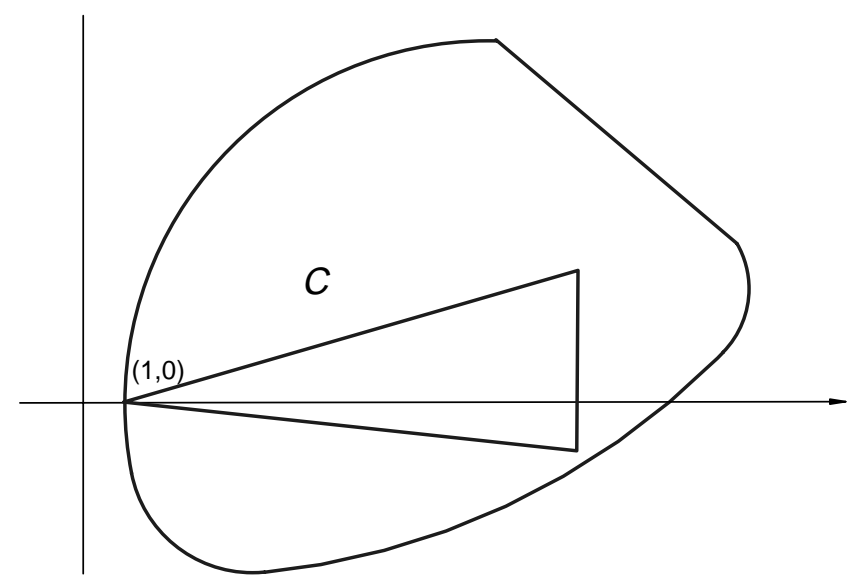

FIGURE 4. 
Then, by (3.9) and (3.10),

$$
\begin{aligned}
& \int_{\mathbb{R}} \int_{\mathbb{R}}|\widehat{g}(\xi, \eta)| d \xi d \eta \\
& =\int_{\mathbb{R}} \int_{\mathbb{R}}\left|\int_{\mathbb{R}}\left\{\int_{\mathbb{R}} g(x, y) e^{-2 \pi i \eta y} d y\right\} e^{-2 \pi i \xi x} d x\right| d \xi d \eta \\
& \geq c \int_{\mathbb{R}} \int_{1}^{+\infty} \frac{1}{x}\left|\int_{\mathbb{R}} g(x, y) e^{-2 \pi i \eta y} d y\right| d x d \eta \\
& \geq c \int_{1}^{r} \frac{1}{x} \int_{\mathbb{R}}\left|\int_{\mathbb{R}} g(x, y) e^{-2 \pi i \eta y} d y\right| d \eta d x \\
& \geq c \int_{1}^{r} \frac{1}{x} \log x d x \\
& =c \log ^{2} r,
\end{aligned}
$$

since, because of the convexity of $B$, we can assume that $g(x, y)$ takes value 1 inside a whole triangle such as the one in the previous picture.

Proof of Theorem 10. Arguing by contradiction, we assume the existence of a positive continuous function $\varepsilon(\rho) \rightarrow 0$ (as $\rho \rightarrow+\infty)$ such that

$$
\int_{0}^{2 \pi}\left|\widehat{\chi}_{B}(\rho \Theta)\right| d \theta \leq \varepsilon(\rho) \rho^{-2} \log \rho
$$

for $\rho \geq 2$. Let $\varphi$ be a nonnegative radial cutoff function supported in the unit disc; then the convolution

$$
g=\chi_{\rho B} * \varphi
$$

satisfies the assumptions in the previous lemma ( $\rho B$ contains a disc of radius $\approx \rho$ ). Therefore, by (3.11),

$$
\begin{aligned}
\log ^{2} \rho & \leq c\|\widehat{g}\|_{L^{1}\left(\mathbb{R}^{2}\right)} \\
& =c \rho^{2} \int_{\mathbb{R}^{2}}\left|\widehat{\chi}_{B}(\rho x) \widehat{\varphi}(x)\right| d x \\
& \leq c \rho^{2} \int_{\mathbb{R}^{2}}\left|\widehat{\chi}_{B}(\rho x)\right| \frac{1}{1+|x|} d x \\
& \leq c \rho^{2} \int_{0}^{+\infty} \frac{u}{1+u} \int_{0}^{2 \pi}\left|\widehat{\chi}_{B}(\rho u \Theta)\right| d \theta d u \\
& =c \int_{0}^{+\infty} \frac{s}{1+\rho^{-1} s} \int_{0}^{2 \pi}\left|\widehat{\chi}_{B}(s \Theta)\right| d \theta d s \\
& \leq c\left(1+\int_{2}^{+\infty} \frac{\varepsilon(s) \log s}{s\left(1+\rho^{-1} s\right)} d s\right) \\
& \leq c\left(1+\int_{2}^{\rho} \frac{\varepsilon(s) \log s}{s} d s+\rho \int_{\rho}^{+\infty} \frac{\varepsilon(s) \log s}{s^{2}} d s\right) \\
& =A(\rho) .
\end{aligned}
$$


To end the proof we observe that

$$
\frac{A(\rho)}{\log ^{2} \rho} \rightarrow 0
$$

as $\rho \rightarrow+\infty$, by l'Hôpital's rule.

Remark 22. Using an induction argument as in [27], the above theorem can be extended to several variables, so that, for any convex body in $\mathbb{R}^{n}$,

$$
\limsup _{\rho \rightarrow+\infty} \frac{\rho^{n}}{\log ^{n-1} \rho} \int_{\Sigma_{n-1}}\left|\widehat{\chi}_{B}(\rho \sigma)\right| d \sigma>0 .
$$

Remark 23. To prove our theorem we have used an idea introduced in [27] to get lower bounds for Lebesgue constants. Therefore our result shows a relation between the study of Lebesgue constants and the $L^{1}$ spherical averages of Fourier transforms of characteristic functions. However, we see no general theorem relating one to the other. See [18] for a related discussion with a number-theoretic flavor.

Remark 24. Estimating $|r(B, \delta, \theta)|$ (see (1.5)) is a geometrical problem which does not necessarily involve the Fourier transform. The previous theorem and the inequality in Lemma [17)imply that, for any convex planar body,

$$
\limsup _{\delta \rightarrow 0^{+}} \frac{1}{\delta \log (1 / \delta)} \int_{0}^{2 \pi}|r(B, \delta, \theta)| d \theta>0 .
$$

The problem considered in the previous remark could be related to the study of floating bodies (see e.g. [22]), where, in place of fixing $\delta$, one fixes the area

$$
(\approx \delta|r(B, \delta, \theta)|)
$$

of the small part of $B$ cut away by the chord $r(B, \delta, \theta)$ in the direction $\Theta$.

Proof of Theorem [1] Arguing as in [15] or 7] and applying Theorem 2] and (1.2), we have

$$
\begin{aligned}
& \int_{\mathbb{T}^{2}} \int_{S O(2)}\left|D_{\rho}\left(\sigma^{-1}(B)-t\right)\right| d \sigma d t \\
& =\rho^{2} \int_{\mathbb{T}^{2}} \int_{S O(2)}\left|\sum_{m \neq 0} \widehat{\chi}_{B}(\rho \sigma m) e^{2 \pi i m \cdot t}\right| d \sigma d t \\
& \leq \rho^{2} \int_{\mathbb{T}^{2}} \int_{S O(2)}\left|\sum_{0 \neq|m| \leq \rho^{(1-2 \alpha) /(1+2 \alpha)}} \widehat{\chi}_{B}(\rho \sigma m) e^{2 \pi i m \cdot t}\right| d \sigma d t \\
& +\rho^{2} \int_{\mathbb{T}^{2}} \int_{S O(2)}\left|\sum_{|m|>\rho^{(1-2 \alpha) /(1+2 \alpha)}} \widehat{\chi}_{B}(\rho \sigma m) e^{2 \pi i m \cdot t}\right| d \sigma d t \\
& \leq \rho^{2} \int_{0 \neq|m| \leq \rho^{(1-2 \alpha) /(1+2 \alpha)}}\left|\widehat{\chi}_{B}(\rho \sigma m)\right| d \sigma(2) \\
& \quad+\rho^{2}\left\{\int_{S O(2)} \sum_{|m|>\rho^{(1-2 \alpha) /(1+2 \alpha)}}\left|\widehat{\chi}_{B}(\rho \sigma m)\right|^{2} d \sigma\right\}^{1 / 2}
\end{aligned}
$$




$$
\begin{aligned}
& \lesssim \rho^{2} \sum_{0 \neq|m| \leq \rho^{(1-2 \alpha) /(1+2 \alpha)}}|\rho m|^{-2+\alpha} \log |\rho m| \\
& +\rho^{2}\left\{\sum_{|m|>\rho^{(1-2 \alpha) /(1+2 \alpha)}}|\rho m|^{-3}\right\}^{1 / 2} \\
& \lesssim \rho^{\alpha} \int_{1}^{\rho^{(1-2 \alpha) /(1+2 \alpha)}} t^{\alpha-1} \log (\rho t) d t+\rho^{1 / 2}\left\{\int_{\rho^{(1-2 \alpha) /(1+2 \alpha)}}^{+\infty} t^{-2}\right\}^{1 / 2} \\
& \lesssim \rho^{2 \alpha /(1+2 \alpha)} .
\end{aligned}
$$

The lower bound follows from Theorem 2 and the orthogonality argument in [7] p. 269].

Proof of Theorem 16. We prove only the case $0<\alpha<1 / 2$. Write $N$ as a sum of four squares, $N=j^{2}+k^{2}+\ell^{2}+m^{2}$, and let $a_{1}, a_{2}, a_{3}, a_{4} \in[0,1)$ be pairwise linearly independent on $\mathbb{Z}$, so that, e.g.,

$$
a_{1}+\frac{p}{j} \neq a_{2}+\frac{q}{k}
$$

for any choice of the integers $p, q, j, k(j, k \neq 0)$. That is,

$$
\left(a_{1}+j^{-1} \mathbb{Z}\right) \cap\left(a_{2}+k^{-1} \mathbb{Z}\right)=\emptyset
$$

when $j \neq k$. Let

$$
A_{j^{2}}=\left\{\left(a_{1}+\frac{p}{j}, \frac{q}{j}\right)\right\}_{p, q \in \mathbb{Z}} \cap \mathbb{T}^{2}
$$

and let us define $A_{k^{2}}, A_{\ell^{2}}, A_{m^{2}}$ accordingly. Define

$$
\mathcal{P}=A_{j^{2}} \cup A_{k^{2}} \cup A_{\ell^{2}} \cup A_{m^{2}} .
$$

By (3.12) $\mathcal{P}$ has cardinality $N$. Since

$$
\begin{aligned}
& \operatorname{card}(\mathcal{P} \cap B)-N|B| \\
& =\operatorname{card}\left(A_{j^{2}} \cap B\right)-j^{2}|B|+\ldots+\operatorname{card}\left(A_{m^{2}} \cap B\right)-m^{2}|B|,
\end{aligned}
$$

it is enough to prove that, say,

$$
\int_{\mathbb{T}^{2}} \int_{S O(2)}\left|\operatorname{card}\left(A_{j^{2}} \cap(\sigma(B)+t)\right)-j^{2}\right| B|| d \theta d t \lesssim N^{\frac{\alpha}{1+2 \alpha}} \log N .
$$

We can therefore prove the theorem assuming $N$ to be a square, say $N=r^{2}, r \in \mathbb{N}$, and

$$
\mathcal{P}=A_{N}=\left\{\left(a+\frac{p}{r}, \frac{q}{r}\right)\right\}_{p, q \in \mathbb{Z}^{2}} \cap U .
$$

Now observe that, writing $w=(a, 0)$ and applying Theorem [1], we have 


$$
\begin{aligned}
& \int_{\mathbb{T}^{2}} \int_{S O(2)}|D(\mathcal{P}, \theta, t)| d t d \sigma \\
& =\int_{S O(2)} \int_{\mathbb{T}^{2}}\left|\operatorname{card}\left(A_{r^{2}} \cap(\sigma(B)+t)\right)-r^{2}\right| B|| d t d \sigma \\
& =\int_{S O(2)} \int_{\mathbb{T}^{2}}\left|\operatorname{card}\left(A_{r^{2}} \cap(\sigma(B)+t+w)\right)-r^{2}\right| B|| d t d \sigma \\
& =\int_{S O(2)} \int_{\mathbb{T}^{2}}\left|\operatorname{card}\left(\left\{\left(\frac{p}{r}, \frac{q}{r}\right)\right\}_{p, q=0}^{r-1} \cap(\sigma(B)+u)\right)-r^{2}\right| B|| d u d \sigma \\
& =\int_{S O(2)} \int_{\mathbb{T}^{2}}\left|\operatorname{card}\left(\mathbb{Z}^{2} \cap(r \sigma(B)+r u)\right)-r^{2}\right| B|| d u d \sigma \\
& =\int_{S O(2)} \int_{\mathbb{T}^{2}}\left|\operatorname{card}\left(\mathbb{Z}^{2} \cap(r \sigma(B)+u)\right)-r^{2}\right| B|| d u d \sigma \\
& \lesssim r^{2 \alpha /(1+2 \alpha)} \log r \\
& =\frac{1}{2} N^{\alpha /(1+2 \alpha)} \log N,
\end{aligned}
$$

where we have used the fact that, for a function $f \in L^{1}\left(\mathbb{T}^{2}\right)$ and for any integer $k \neq 0$,

$$
\int_{\mathbb{T}^{2}} f(k u) d u=\int_{\mathbb{T}^{2}} f(u) d u
$$

The above argument extends to several variables after replacing the sum of four squares by Hilbert's theorem (Waring's problem).

\section{REFERENCES}

[1] J. Beck, Irregularities of distribution I, Acta Math. 159 (1987), 1-49. MR 89c:11117

[2] J. Beck and W.W.L. Chen, Irregularities of distribution, Cambridge University Press, 1987. MR 88m:11061

[3] J. Beck and W.W.L. Chen, Note on irregularities of distribution II, Proc. London Math. Soc. 61(1990), 251-272. MR 91g:11083

[4] J. Beck and W.W.L. Chen, Irregularities of point distribution relative to convex polygons II, Mathematika 40 (1993), 127-136. MR 94i:11055

[5] L. Brandolini and L. Colzani, Localization and convergence of eigenfunction expansions, Journal Fourier Anal. Appl. 5 (1999), 431-447. MR 2001g:42054

[6] L. Brandolini and L. Colzani, Decay of Fourier Transforms and Summability of Eigenfunction Expansions, Ann. Scuola Norm. Sup. Pisa Cl. Sci (4) 29 (2000), 611-638. MR 2002e:35178

[7] L. Brandolini, L. Colzani and G. Travaglini, Average decay of Fourier transforms and integer points in polyhedra, Ark. Mat. 35 (1997), 253-275. MR 99e:42021

[8] L. Brandolini, M. Rigoli and G. Travaglini, Average decay of Fourier transforms and geometry of convex sets, Revista Mat. Iberoamericana 14 (1998), 519-560. MR 2000a:42017

[9] L. Brandolini and G. Travaglini, Pointwise convergence of Fejer type means, Tohoku Math. J. 49 (1997), 323-336. MR 98m:42010

[10] J. Bruna, A. Nagel and S. Wainger, Convex hypersurfaces and Fourier transforms, Ann. Math. 127 (1988), 333-365. MR 89d:42023

[11] W.W.L. Chen, On irregularities of distribution III, J. Austr. Math. Soc. 60 (1996), 228-244. MR 97e:11085

[12] R. Coifman and G. Weiss, Extensions of Hardy spaces and their use in analysis, Bull. Amer. Math. Soc. 83 (1977), 569-645. MR 56:6264 
[13] L. Colzani, Fourier transform of distributions in Hardy spaces, Boll. Un. Mat. Ital. 1 (1982), 403-410. MR 84c:46042

[14] H. Davenport, Note on irregularities of distribution, Mathematika 1 (1954), 73-79. MR 18:566a

[15] D. G. Kendall, On the number of lattice points inside a random oval, Quart. J. Math. Oxford Ser. 19 (1948), 1-26. MR 9:570b

[16] E. Krätzel, Lattice points, Kluwer Academic Publisher, 1988. MR 90e:11144

[17] H. L. Montgomery, Ten lectures on the interface between analytic number theory and harmonic analysis, CBMS Regional Conference Series in Mathematics, 84, American Mathematical Society, Providence, RI, 1994. MR 96i:11002

[18] F. L. Nazarov and A. N. Podkorytov, On the behaviour of the Lebesgue constants for twodimensional Fourier sums over polygons, St. Petersburg Math. J. 7 (1996), 663-680. MR 96m:42019

[19] A. N. Podkorytov, On the asymptotics of the Fourier transform on a convex curve, Vestn. Leningrad Univ. Math. 24 (1991), no. 2, 57-65. MR 93h:42019

[20] B. Randol, On the Fourier transform of the indicator function of a planar set, Trans. Amer. Math. Soc. 139 (1969), 271-278. MR 40:4678a

[21] F. Ricci and G. Travaglini, Convex curves, Radon transforms and convolution operators defined by singular measures, Proc. Amer. Math. Soc. 129 (2001), 1739-1744. MR 2002i:42010

[22] C. Schütt, The convex floating body and polyhedral approximation, Israel J. Math. 73 (1991), 65-77. MR 92i:52009

[23] A. Seeger and S. Ziesler, Riesz means associated with convex domains in the plane. Math. Z. 236 (2001), 643-676. MR 2002j:42011

[24] W. T. Sledd and D. A. Stegenga, An $H^{1}$ multiplier theorem. Ark. Mat. 19 (1981), 265-270. MR 84j:42018

[25] M. Tarnopolska-Weiss, On the number of lattice points in a compact n-dimensional polyhedron, Proc. Amer. Math. Soc. 74 (1979), 124-127. MR 80c:10048

[26] A. A. Yudin and V. A. Yudin, Polygonal Dirichlet kernels and growth of Lebesgue constants. Mat. Zametki 37 (1985), 220-236; English transl., Math. Notes 37 (1985), 124-135. MR 86k:42039

[27] V. A. Yudin, Lower bound for Lebesgue constants, Mat. Zametki 25 (1979), 119-122; English transl., Math. Notes 25 (1979), 63-65. MR 80i:42010

Dipartimento di Ingegneria, Università di Bergamo, Viale G. Marconi 5, 24044 Dalmine (BG), Italy

E-mail address: brandolini@unibg.it

Department of Mathematics, University of Missouri, Columbia, Missouri

E-mail address: iosevich@math.missouri.edu

URL: http://www.math.missouri.edu/〜iosevich/

Dipartimento di Matematica e Applicazioni, Università di Milano-Bicocca, Via Bicocca Degli Arcimboldi 8, 20126 Milano, Italy

E-mail address: travaglini@matapp.unimib.it

$U R L:$ http://www.matapp.unimib.it/ travaglini/ 